\title{
Core in a simple coalition formation game
}

\author{
Suryapratim Banerjee $^{1}$, Hideo Konishi ${ }^{2}$, Tayfun Sönmez ${ }^{3}$ \\ ${ }^{1}$ Department of Economics, McGill University, Montreal, Canada H3A 2T7 \\ ${ }^{2}$ Department of Economics, Boston College, Chestnut Hill, MA 02467, USA \\ ${ }^{3}$ University of Michigan and Department of Economics, Koç University Istinye, \\ 80860, Istanbul, Turkey
}

Received: 31 July 1999/Accepted: 5 January 2000

\begin{abstract}
We analyze the core of a class of coalition formation game in which every player's payoff depends only on the members of her coalition. We first consider anonymous games and additively separable games. Neither of these strong properties guarantee the existence of a core allocation, even if additional strong properties are imposed. We then introduce two top-coalition properties each of which guarantee the existence. We show that these properties are independent of the Scarf-balancedness condition. Finally we give several economic applications.
\end{abstract}

\section{Introduction}

Non-emptiness of the core in coalition formation games has been analyzed by several authors including Guesnerie and Oddou (1981), Greenberg and

We thank Anna Bogomolnaia, John Conley, Rajat Deb, Bhaskar Dutta, Hsueh-Ling Huynh, Matthew Jackson, Michel Le Breton, Glenn Loury, Dilip Mookherjee, Tom Quint, Debraj Ray, Koichi Tadenuma, Rajiv Vohra, Jun Wako, Shlomo Weber and the seminar participants at Duke University, Hitotsubashi University, University of British Columbia, the Fourth International Meeting of the Society of Social Choice and Welfare in Vancouver, and the Congress on "Groups, Networks and Coalitions" in Manresa. We are especially indebted to Nadia Burani, Hervé Moulin, William Thomson, Lin Zhou, William Zwicker and an anonymous referee for their helpful comments. This research was conducted while Konishi was visiting Boston University and Kyoto Institute of Economic Research. He is grateful to their hospitality and financial support. Sönmez gratefully acknowledges the research support of the National Science Foundation via grants SBR-9709138 and SES-9904214. All errors are our own responsibility. 
Weber (1986), Demange (1994) in local public goods context and Greenberg and Weber (1993) in political economy context. In this paper, we formulate a general class of coalition formation problems and study core existence in this class. We assume away alternatives available to each coalition and focus on purely hedonic aspect of coalition formation: A coalition formation game is a finite player game in which each player's payoff depends only on the members of her coalition (no spillovers: outsiders' behavior does not affect the coalition members' payoffs). ${ }^{1}$ A feasible allocation is a partition of the players. There is no transferrable money. Our model is closely related to matching problems where preferences are also hedonic but not all coalition structures are feasible. ${ }^{2}$ We choose the core (the coalition structure core) as our solution concept. A core partition is stable in the sense that there is no profitable coalitional deviation.

Our model can be regarded as a reduced two-stage game, where players first form coalitions and next negotiate their payoffs inside coalitions. Once the second step is solved, we are left with the situation described by our hedonic model.

Unfortunately, without any restriction on preferences the core may be empty. Consider the following example: There are three students in a college who are trying to choose their roommates. There are only two-bedroom apartments available on campus and these apartments are fairly expensive. So each student wants to share an apartment rather than to live alone, but sharing with two others is the last choice since one of them would have to sleep in the living room. Suppose that student 1 likes student 2 better than student 3 , student 2 likes student 3 better than student 1 , and student 3 likes student 1 better than student 2 . Is there a stable roommate structure (a core partition) in this situation? Suppose that there is one. Since sharing with two others is the last choice for all of them, there should be at least one student who lives alone at any core partition. Who can this student be? She cannot be student 1 since she can easily convince student 3 to share an apartment. Similarly student 2 can easily convince student 1 and student 3 can easily convince student 2. Hence, there is no core partition in this example.

Although this game is very simple, it does not have a core partition. The key problem is cyclical coalitional deviations. A natural question is whether we can avoid these cycles by imposing some natural properties such as anonymity and additive separability. Anonymity requires agents not to care for identities of their partners and thus only care for the coalition size. Additive separability requires the preferences to be representable by an additively separable utility function. Note that our motivating example lacks both properties. We investigate the existence of a core partition by imposing both properties separately. Unfortunately neither of these conditions guarantee existence even if we impose additional strong conditions such as single-peakedness and

\footnotetext{
1 This model is independently introduced by Bogomolnaia and Jackson (1998).

2 See Roth and Sotomayor (1990) for an excellent survey of matching problems.
} 
the intermediate preference property. In order to prove non-emptiness of the core, the single-peakedness and the intermediate preference property have been employed by Greenberg and Weber (1993) and in Greenberg and Weber (1986), Demange (1994) respectively. Those properties are powerful tools in games without congestion, ${ }^{3}$ but they turn out to be inessential in games with possible congestion. (See Games 1 and 3.)

We next introduce the top-coalition property which imposes a commonality of preferences among the players. This condition requires that for any non-empty subset $S$ of players, we can find a coalition $T \subseteq S$ such that all members of $T$ prefer $T$ to any other coalition that consists of some (or all) members of $S$. This property is a relaxed version of the common ranking property (Farrell and Scotchmer 1988). The common ranking property requires that there is a linear ordering over all coalitions which coincides with any player's preference ordering over coalitions to which she belongs. Although both properties guarantee the non-emptiness of the core, the topcoalition property is weaker and hence it covers more games. Indeed, even a weaker version of the top-coalition property guarantees the existence. While the weak top-coalition property is still demanding, it is satisfied in many interesting economic applications. Examples include coalition formation games induced by the average cost sharing rule (Moulin and Shenker 1994) in the context of cost sharing problems, coalition formation games induced by the competitive mechanism in the context of housing markets (Shapley and Scarf 1974), and coalition formation games induced by the uniform reallocation rule (Benassy 1982) in the context of reallocation problems. We also show that these conditions do not imply the Scarf-balancedness (Scarf 1967) condition which is often used to prove the non-emptiness of the core in NTU games.

Three papers are closely related to ours: Bogomolnaia and Jackson (1998) independently introduce coalition formation games and they use core as well as individual stability (see Greenberg 1977 and Dréze and Greenberg 1980), Nash stability, and contractual individual stability as their stability notions. A partition is individually stable if and only if no player wants to move from her coalition to another one (including the empty coalition) that welcomes her unanimously. Thus, individual stability is a less demanding stability concept than the core stability. This notion is particularly appealing if the cost of forming a new coalition is high. Nash stability is a stronger version of individual stability where players do not need any permission to join a new coalition. A partition is contractually individually stable if and only if no player wants to move from her coalition to another one that welcomes her unanimously, without hurting anyone in the old coalition. Bogomolnaia and Jackson study an ordinal version of Scarf's balancedness condition as well as a weak consecutiveness property and show that each ensure the non-emptiness

\footnotetext{
${ }^{3}$ For example, Weber and Zamir (1985) construct a four player example with empty core when the intermediate preference property is violated in an economy analyzed by Guesnerie and Oddou (1981) and Greenberg and Weber (1986).
} 
of the core. ${ }^{4}$ They next show that there exists an individually stable partition under anonymity and single-peakedness, and even a Nash stable partition under additive separability and symmetry. Note that these conditions do not guarantee non-emptiness of the core. (See Games 1 and 2.) They also show that Pareto efficiency implies contractual individual stability and finally analyze the compatibility of these stability notions with several normative criteria. Kaneko and Wooders (1986) show that the core of a coalition formation game is non-empty, provided that (i) there is a continuum of players with finite types and (ii) each coalition is finite in size. Their result dramatically contrasts the core in large games with the core in our finite games. Milchtaich and Winter (1997) introduce a model where (i) the number of coalitions is fixed, (ii) each agent is endowed with a number, and (iii) each agent prefers to be in a group which is most similar to herself on average. They show that a Nash stable partition exists that is segregating and Pareto efficient.

The organization of the rest of the paper is as follows: In Sect. 2 we formally introduce the model. In Sects. 3 and 4 we demonstrate that anonymity and additive separability are inessential for the non-emptiness of the core. In Sect. 5 we present two top-coalition properties and provide an existence result. We also discuss the inclusion relationships between the two top-coalition properties, the common ranking property and the Scarf-balancedness condition. Finally we present several applications in Sect. 6 .

\section{The model}

The following model is independently introduced by Bogomolnaia and Jackson (1998):

Definition 1. A coalition formation game $G$ is a pair $\left(N,\left(\succeq_{i}\right)_{i \in N}\right)$, where $N$ is a finite player set with $i$ being a representative element, and $\succeq_{i}$ is a reflexive, complete, and transitive binary relation on $S_{i}(N)=\left\{S \in 2^{N}: i \in S\right\}$. Strict preference relation and the indifference relation are denoted by $\succ_{i}$ and $\sim_{i}$ respectively ( $S \succ_{i} T \Leftrightarrow\left[S \succeq_{i} T\right.$ and $\left.T \succeq_{i} S\right]$ and $S \sim_{i} T \Leftrightarrow\left[S \succeq_{i} T\right.$ and $\left.T \succeq_{i} S\right]$ ).

Definition 2. A coalition structure $\pi=\left\{S_{1}, S_{2}, \ldots, S_{K}\right\} \quad(K \leq|N|$ is a positive integer) is a partition of $N$. That is, $S_{k} \neq \varnothing$ for any $k \in\{1,2, \ldots, K\}, \bigcup_{k=1}^{K} S_{k}$ $=N$, and $S_{k} \cap S_{\ell}=\varnothing$ for any $k, \ell \in\{1,2, \ldots, K\}$ with $k \neq \ell$. For any coalition structure $\pi$ and any player $i$ let $\pi(i)=\{S \in \pi: i \in S\}$ be the set of her partners. The collection of all coalition structures in $N$ is denoted by $\Pi(N)$. We similarly define $\Pi(V)$ for any $V \subset N$ with $V \neq \varnothing$.

\footnotetext{
${ }^{4}$ A coalition formation game is weakly consecutive if there exists an ordering of agents such that any partition that is not in the core can be blocked by a set of "connected" agents. Our top-coalition property implies the weak consecutiveness property. However there is no inclusion relation between the weak top-coalition property, the weak consecutiveness property, and the ordinal balance property (see Proposition 1 in Bogomolnaia and Jackson 1998).
} 
Definition 3. A coalition $T \in 2^{N} \backslash\{\varnothing\}$ is a (profitable) coalitional deviation from $\pi \in \Pi(N)$ iff $T \succ_{i} \pi(i)$ for any $i \in T$. A core partition is a partition $\pi^{*}$ that is immune to any coalitional deviation.

In the following two sections, we present examples showing that anonymity and additive separability are inessential to the non-emptiness of the core.

\section{Anonymity}

We first consider games in which players only care about the size of their coalitions.

Definition 4. A game $G$ satisfies anonymity iff for any $i \in N$, for any $S, T \in$ $S_{i}(N)$ with $|S|=|T|$ we have $S \sim_{i} T$.

The core may be empty even under anonymity. Indeed, it can be empty even if additional stringent conditions are imposed following Greenberg and Weber $(1986,1993)$ and Demange (1994). ${ }^{5}$

Definition 5. An anonymous game $G$ satisfies single-peakedness on population if for any $i \in N$, and any $S, T, U \in S_{i}(N)$ with $|S|<|T|<|U|$, we have (i) $S \succeq_{i} T \Rightarrow T \succ_{i} U$ and (ii) $U \succeq_{i} T \Rightarrow T \succ_{i} S$.

Definition 6. An anonymous game $G$ satisfies the population intermediate preference property if the players can be linearly ordered by a binary relation $\prec$ in such a way that for any $i \prec j \prec k$, and any $S, T \in \bigcap_{t \in\{i, j, k\}} S_{t}(N),\left[S \succeq_{i} T\right.$ and $\left.S \succeq_{k} T\right] \Rightarrow S \succeq_{j} T$.

Although these restrictions are undoubtedly strong, they do not guarantee the non-emptiness of the core.

Game 1. There are four type- $a$, one type- $b$, and two type-c players. Thus, the player set $N$ can be written as $N=\left\{a_{1}, a_{2}, a_{3}, a_{4}, b_{1}, c_{1}, c_{2}\right\}$. Their preferences are as follows (for simplicity we abuse notation and specify the preferences over cardinalities of coalitions):

5 Greenberg and Weber $(1986,1993)$ and Demange (1994) assume that there is a set of alternatives $A$ on which preferences are defined, and they impose on $A$ either singlepeakedness or the intermediate preference property:

Single-Peakedness (Greenberg and Weber 1993): There is a linear order $\succ$ on $A$ with the following property: For each $i \in N$, there exists an alternative $a_{i}^{*} \in A$ such that (i) for any $a \succ b \succ a_{i}^{*}, a \preceq_{i} b \preceq_{i} a_{i}^{*}$ holds and (ii) for any $a \prec b \prec a_{i}^{*}, a \preceq_{i} b \preceq_{i} a_{i}^{*}$ holds.

The Intermediate Preference Property (Greenberg and Weber 1986, Demange 1994): There is a tree $\mathscr{G}(N)$ defined on the set of players (see the next section) and if player $m$ is on the path between $i$ and $j(i \neq m, m \neq j)$, then for any $a, b \in A$ with $a \neq b$, $\left[a \succeq_{i} b\right.$ and $\left.a \succeq_{j} b\right] \Rightarrow\left[a \succeq_{m} b\right]$.

In both papers the authors define a feasibility correspondence $\varphi: 2^{N} \backslash\{\varnothing\} \rightarrow 2^{A}$, which means that a coalition $S$ can choose an alternative from $\varphi(S)$. The correspondence $\varphi$ is assumed to satisfy monotonicity: For any $S, T \in 2^{N} \backslash\{\varnothing\}$ with $S \subset T$, $\varphi(S) \subseteq \varphi(T)$. Thus, in their games, there is no congestion effect besides possible coordination failures. 
Type a: $6 \succ_{a} 5 \succ_{a} 4 \succ_{a} 3 \succ_{a} 2 \succ_{a} 1 \succ_{a} 7$,

Type $b: 5 \succ_{b} 4 \succ_{b} 3 \succ_{b} 6 \succ_{b} 2 \succ_{b} 1 \succ_{b} 7$,

Type c: $4 \succ_{c} 3 \succ_{c} 5 \succ_{c} 6 \succ_{c} 2 \succ_{c} 1 \succ_{c} 7$.

Note that Game 1 satisfies anonymity, single-peakedness on population, and the population intermediate preference property. ${ }^{6}$

Claim. The core of Game 1 is empty.

Proof of the Claim. Let $k$ be the cardinality of the largest coalition in a coalition structure. We show that there is no coalition structure which forms a core partition by exhausting all cases. First consider all cases with $k \leq 4$. Then coalition $\left\{a_{1}, a_{2}, a_{3}, a_{4}, b_{1}\right\}$ deviates from any such coalition structure. Second, if $k=7$ then every singleton coalition deviates. Third, if $k=6$ then coalition $\left\{b_{1}, c_{1}, c_{2}\right\}$ deviates. Thus, if there is a core partition then $k=5$. There are five representative cases (which are unique up to type composition):

1. $\left\{\left\{a_{1}, a_{2}, a_{3}, a_{4}, b_{1}\right\},\left\{c_{1}, c_{2}\right\}\right\}$ : In this case $\left\{a_{1}, a_{2}, a_{3}, a_{4}, c_{1}, c_{2}\right\}$ deviate.

2. $\left\{\left\{a_{1}, a_{2}, a_{3}, b_{1}, c_{1}\right\},\left\{a_{4}, c_{2}\right\}\right\}$ : In this case $\left\{a_{4}, c_{1}, c_{2}\right\}$ deviate.

3. $\left\{\left\{a_{1}, a_{2}, a_{3}, c_{1}, c_{2}\right\},\left\{a_{4}, b_{1}\right\}\right\}$ : In this case $\left\{a_{4}, b_{1}, c_{1}, c_{2}\right\}$ deviate.

4. $\left\{\left\{a_{1}, a_{2}, a_{3}, a_{4}, c_{1}\right\},\left\{b_{1}, c_{2}\right\}\right\}$ : In this case $\left\{b_{1}, c_{1}, c_{2}\right\}$ deviate.

5. $\left\{\left\{a_{1}, a_{2}, b_{1}, c_{1}, c_{2}\right\},\left\{a_{3}, a_{4}\right\}\right\}$ : In this case $\left\{a_{3}, a_{4}, c_{1}, c_{2}\right\}$ deviate.

Hence, there is no core partition in this game.

\section{Additive separability}

We next consider games where players have additively separable preferences.

Definition 7. A game $G$ is separable iff for any $i \in N$, for any $S \in S_{i}(N)$ and for any $j \notin S$, we have $\left[S \cup\{j\} \succeq_{i} S \Leftrightarrow\{i, j\} \succeq_{i}\{i\}\right]$ and $\left[S \cup\{j\} \preceq_{i} S \Leftrightarrow\{i, j\} \preceq_{i}\right.$ $\{i\}]$.

Definition 8. A game $G$ is additively separable iff for any $i \in N$, there exists a function $v_{i}: N \rightarrow \mathbb{R}$ such that for any $S, T \in S_{i}(N), S \succeq_{i} T \Leftrightarrow \sum_{j \in S} v_{i}(j) \geq$ $\sum_{k \in T} v_{i}(k)$.

Additive separability is a very strong notion of separability on preferences. Despite this, it does not guarantee the non-emptiness of the core even with additional strong conditions.

Definition 9. A game $G$ satisfies mutuality iff for any $i, j \in N$, we have $\left[\{i, j\} \succeq_{i}\right.$ $\left.\{i\} \Leftrightarrow\{i, j\} \succeq_{j}\{j\}\right]$ and $\left[\{i, j\} \preceq_{i}\{i\} \Leftrightarrow\{i, j\} \preceq_{j}\{j\}\right]$.

\footnotetext{
${ }^{6}$ The population intermediate property is satisfied for the following linear ordering of agents:

$a_{1} \succ a_{2} \succ a_{3} \succ a_{4} \succ b_{1} \succ c_{1} \succ c_{2}$
} 
Mutuality requires that if player $i$ likes $j$ then player $j$ likes $i$ and if player $i$ dislikes $j$ then player $j$ dislikes $i$. We need additional notation in order to define the last two conditions in this section.

A (nondirected) graph $\mathscr{G}$ on $N$ is a set of unordered pairs of distinct elements of $N$. A pair $(i, j) \in \mathscr{G}$ is called an edge between $i$ and $j$. A path of $\mathscr{G}$ is a sequence $i_{1}, i_{2}, \ldots, i_{s}$ where the pairs $\left(i_{k}, i_{k+1}\right)$ are edges for $k=1,2, \ldots, s-1$ and are all distinct. A graph $\mathscr{G}$ is connected if any two distinct elements of $N$ are linked by a path. A graph $\mathscr{G}$ is a tree if any two distinct elements are linked by a unique path. A tree $\mathscr{G}$ is star-shaped if every edge contains the same element of $N$.

Definition 10. A game $G$ satisfies single-peakedness on a tree iff for any $i \in N$, any tree $\mathscr{G}$ on $N$, any path $i_{1}, i_{2}, \ldots, i_{s}$ on $\mathscr{G}$ emanating from $i\left(i_{1}=i\right)$, and any $1<k<\ell \leq s$, we have $\left\{i, i_{k}\right\} \succeq_{i}\left\{i, i_{\ell}\right\} .{ }^{7}$

Definition 11. A game $G$ satisfies the tree intermediate preference property iff for any $i \in N$, any tree $\mathscr{G}$ on $N$, any path $i_{1}, i_{2}, \ldots, i_{s}$ on $\mathscr{G}$, any $1 \leq k<\ell<$ $m \leq s$, and any $j \in N \backslash\left\{i_{k}, i_{\ell}, i_{m}\right\}$, we have $\left[\left\{i_{k}, j\right\} \succeq_{i_{k}}\left\{i_{k}\right\}\right.$ and $\left.\left\{i_{m}, j\right\} \succeq_{i_{m}}\left\{i_{m}\right\}\right]$ $\Rightarrow\left\{i_{\ell}, j\right\} \succeq_{i_{\ell}}\left\{i_{\ell}\right\} .^{8}$

The following game satisfies additive separability and mutuality: ${ }^{9}$

Game 2. $N=\{1,2,3,4,5,6\}$ and the additively separable preferences are summarized by the following table $\left(v_{i}(j)\right)$ :

\begin{tabular}{|c|c|c|c|c|c|c|}
\hline & 1 & 2 & 3 & 4 & 5 & 6 \\
\hline$v_{1}$ & 0 & 3 & 2 & -10 & 2 & 2.5 \\
\hline$v_{2}$ & 3 & 0 & 2.5 & -6 & -10 & -6 \\
\hline$v_{3}$ & 2 & 2.5 & 0 & 3 & 2 & -10 \\
\hline$v_{4}$ & -10 & -6 & 3 & 0 & 2.5 & -6 \\
\hline$v_{5}$ & 2 & -10 & 2 & 2.5 & 0 & 3 \\
\hline$v_{6}$ & 2.5 & -6 & -10 & -6 & 3 & 0 \\
\hline
\end{tabular}

7 Single-peakedness on a tree is considered in Demange (1982) in a voting (public goods) problem.

${ }^{8}$ Our tree intermediate preference property is one-sided in the sense that we do not restrict players' preferences when they dislike another player. A game $G$ satisfies the two-sided tree intermediate preference property iff for any $i \in N$, for any tree $\mathscr{G}$ on $N$, for any path $i_{1}, i_{2}, \ldots, i_{s}$ on $\mathscr{G}$, for any $1 \leq k<\ell<m \leq s$, for any $j \in N \backslash\left\{i_{k}, i_{\ell}, i_{m}\right\}$, (i) $\left[\left\{i_{k}, j\right\} \succeq_{i_{k}}\left\{i_{k}\right\}\right.$ and $\left.\left\{i_{m}, j\right\} \succeq_{i_{m}}\left\{i_{m}\right\}\right] \Rightarrow\left\{i_{\ell}, j\right\} \succeq_{i_{\ell}}\left\{i_{\ell}\right\}$ and (ii) $\left[\left\{i_{k}, j\right\} \preceq_{i_{k}}\left\{i_{k}\right\}\right.$ and $\left.\left\{i_{m}, j\right\} \preceq_{i_{m}}\left\{i_{m}\right\}\right] \Rightarrow\left\{i_{\ell}, j\right\} \preceq_{i_{\ell}}\left\{\bar{i}_{\ell}\right\}$. Game 3 below does not satisfy the two-sided tree intermediate preference property. It is an open question whether two-sidedness would help us obtain a positive result.

9 Indeed, Game 2 satisfies symmetry: For any $i, j \in N, v_{i}(j)=v_{j}(i)$. Clearly symmetry is stronger than mutuality. 
Claim. The core of Game 2 is empty.

Proof of the Claim. Note that no pair of even players can be in the same coalition, since otherwise their payoffs would be lower than standing alone. Note also that player $i$ and player $\bmod _{6}(i+3)$ (the opposite player on a circle) can not be in the same coalition by the same reason. Thus, there are only a few candidates for a core partition:

1. $\{\{1,2,3\},\{4\},\{5,6\}\}$ and its finer refinements: In these cases $\{3,4,5\}$ deviate.

2. $\{\{1,2,3\},\{4,5\},\{6\}\}$ and its finer refinements: In these cases $\{5,6\}$ deviate.

3. $\{\{1,2\},\{3,4,5\},\{6\}\}$ and its finer refinements: In these cases $\{5,6,1\}$ deviate.

4. $\{\{6,1\},\{2\},\{3,4,5\}\}$ and its finer refinements: In these cases $\{1,2\}$ deviate.

5. $\{\{5,6,1\},\{2\},\{3,4\}\}$ and its finer refinements: In these cases $\{1,2,3\}$ deviate.

6. $\{\{5,6,1\},\{2,3\},\{4\}\}$ and its finer refinements: In these cases $\{3,4\}$ deviate.

7. $\{\{1,3,5\},\{2\},\{4\},\{6\}\}$ and its finer refinements: In these cases $\{1,2,3\}$ deviate.

Hence there is no core partition.

The following game, which builds on Game 2, not only satisfies mutuality and additive separability, but also single-peakedness on a tree and the tree intermediate preference property.

Game 3. $N=\{1,2,3,4,5,6,7,8\}$ and the additively separable preferences are summarized by the following table:

\begin{tabular}{|c|c|c|c|c|c|c|c|c|}
\hline & 1 & 2 & 3 & 4 & 5 & 6 & 7 & 8 \\
\hline$v_{1}$ & 0 & 3 & 2 & -10 & 2 & 2.5 & 5 & -20 \\
\hline$v_{2}$ & 3 & 0 & 2.5 & -6 & -10 & -6 & 5 & -20 \\
\hline$v_{3}$ & 2 & 2.5 & 0 & 3 & 2 & -10 & 5 & -20 \\
\hline$v_{4}$ & -10 & -6 & 3 & 0 & 2.5 & -6 & 5 & -20 \\
\hline$v_{5}$ & 2 & -10 & 2 & 2.5 & 0 & 3 & 5 & -20 \\
\hline$v_{6}$ & 2.5 & -6 & -10 & -6 & 3 & 0 & 5 & -20 \\
\hline$v_{7}$ & 5 & 5 & 5 & 5 & 5 & 5 & 0 & 100 \\
\hline$v_{8}$ & -20 & -20 & -20 & -20 & -20 & -20 & 100 & 0 \\
\hline
\end{tabular}

The restriction of this game to $\{1,2,3,4,5,6\}$ is Game 2. We add two more players 7 and 8. We complete the preference specification as follows: Everyone likes player 7 the most but player 8 is "hated" by anybody except player 7. Finally players 7 and 8 "love" each other. 
Claim. Game 3 satisfies additive separability, mutuality, the tree single-peaked property, and the tree intermediate preference property. Nonetheless its core is empty.

Proof of the Claim. It is easy to see that Game 3 satisfies all the conditions. The tree intermediate preference property can be checked by using a star-shaped tree $\mathscr{G}=\{(i, 7): i \in N \backslash\{7\}\}$. We will show that any coalition structure in core must contain $\{7,8\}$ as a component. It is easy to see that none of the first six players can be in the same coalition with player 8 . They would rather be alone than having player 8 as a partner. It is also easy to see that players 7 and 8 should be partners since either player is more valuable for the other than the sum of everyone else. Therefore the coalition $\{7,8\}$ is a component of any core partition. But since (i) the restriction of Game 3 to remaining players is Game 2 and (ii) Game 2 has an empty core, Game 3 has an empty core as well.

\section{Two top-coalition properties}

Farrell and Scotchmer (1988) show that the following condition guarantees the non-emptiness of the core:

Definition 12. (Farrell and Scotchmer 1988) A game G satisfies the common ranking property iff there exists an ordering $\succeq$ over $2^{N} \backslash\{\varnothing\}$ such that for any $i \in N$ and any $S, T \in S_{i}(N)$ we have $S \succeq_{i} T \Leftrightarrow S \succeq T$.

Next consider the following relaxation of the common ranking property.

Definition 13. Given a non-empty set of players $V \subseteq N$, a non-empty subset $S \subseteq V$ is a top-coalition of $V$ iff for any $i \in S$ and any $T \subseteq V$ with $i \in T$, we have $S \succeq_{i} T$. A coalition formation game $G$ satisfies the top-coalition property iff for any non-empty set of players $V \subseteq N$, there exists a top-coalition of $V$.

This condition requires that, for any group of players $V$ there is a subgroup that is mutually the best for all its members. The top-coalition property reduces to $\alpha$-reducibility (Alcalde 1995) in roommate problems (Gale and Shapley 1962). ${ }^{10}$ We finally have a positive result with the top-coalition property. Indeed, even the following weaker version guarantees the existence.

Definition 14. Given a non-empty set of players $V \subseteq N$, a non-empty subset $S \subseteq V$ is a weak top-coalition of $V$ iff $S$ has an ordered partition $\left\{S^{1}, \ldots, S^{l}\right\}$ such that (i) for any $i \in S^{1}$ and any $T \subseteq V$ with $i \in T$, we have $S \succeq_{i} T$ and (ii) for any $k>1$, any $i \in S^{k}$, and any $T \subseteq V$ with $i \in T$, we have $T \succ_{i} S \Rightarrow T \cap$ $\left(\bigcup_{m<k} S^{m}\right) \neq \varnothing$. A coalition formation game $G$ satisfies the weak top-coalition property iff for any non-empty set of players $V \subseteq N$, there exists a weak topcoalition of $V$.

10 Tan (1991) provides a necessary and sufficient condition for the non-emptiness of the core for roommate problems. 
A coalition $S$ is a weak top coalition of $V$ if it has a partition $\left\{S^{1}, \ldots, S^{l}\right\}$ such that:

1. Any agent in $S^{1}$ prefers $S$ to any subset of $V$,

2. any agent in $S^{2}$ needs cooperation of at least one agent in $S^{1}$ in order to form a better coalition than $S$,

3. any agent in $S^{3}$ needs cooperation of at least one agent in $S^{1} \cup S^{2}$ in order to form a better coalition than $S$,

l. any agent in $S^{l}$ needs cooperation of at least one agent in $\bigcup_{m<l} S^{m}$ in order to form a better coalition than $S$.

Theorem 1. Suppose game G satisfies the weak top-coalition property. Then it has a non-empty core. ${ }^{11}$

Proof. Let $V_{0}=N, S_{1} \subseteq V_{0}$ be a weak top-coalition of $V_{0}$, and $\left\{S_{1}^{1}, \ldots, S_{1}^{l(1)}\right\}$ be its partition as defined in Definition 14. Let $V_{1}=V_{0} \backslash S_{1}, S_{2} \subseteq V_{1}$ be a weak top-coalition of $V_{1}$, and $\left\{S_{2}^{1}, \ldots, S_{2}^{l(2)}\right\}$ be its partition as defined in Definition 14. Similarly, define the triple $\left(S_{k},\left\{S_{k}^{1}, \ldots, S_{k}^{l(k)}\right\}, V_{k}\right)$ for each $k$. Since the set of players $N$ is finite, this procedure terminates in finite steps (i.e., there is an integer $K$ such that $V_{K}=\varnothing$ and $\left.V_{K-1} \neq \varnothing\right)$. Let $\pi^{*}=\left\{S_{1}, \ldots, S_{K}\right\}$. We show that the coalition structure $\pi^{*}$ is a core partition. Consider the following sequence of groups: $S_{1}^{1}, \ldots, S_{1}^{l(1)}, S_{2}^{1}, \ldots, S_{2}^{l(2)}, \ldots, S_{K}^{1}, \ldots, S_{K}^{l(K)}$. Observe that no agent in $S_{1}^{1}$ could profit from joining a coalitional deviation. Without the help of agents in $S_{1}^{1}$, no agent in $S_{1}^{2}$ could profit from joining a coalitional deviation. In general, without the help of agents from earlier groups in the sequence, a profitable coalitional deviation is not possible. Hence there is no profitable coalitional deviation and $\pi^{*}$ is in the core.

Moreover, the core is a singleton provided that the top-coalition property is satisfied and the preferences are strict.

Theorem 2. Suppose game G satisfies the top-coalition property and the preferences are strict. Then it has a unique core partition.

Proof. For any $V \subseteq N$ define $M(V)=\left\{S \in 2^{V} \backslash \varnothing: S\right.$ is a top-coalition of $V\}$. Under strict preferences $M(V)$ is a collection of disjoint sets for any nonempty $V \subseteq N$. Define $V_{0}=N$ and let $M\left(V_{0}\right)=\left\{S_{1}^{1}, \ldots, S_{1}^{m(1)}\right\}$. Next define $V_{1}=N \backslash \bigcup_{j=1}^{m(1)} S_{1}^{j}$ and let $M\left(V_{1}\right)=\left\{S_{2}^{1}, \ldots, S_{2}^{m(2)}\right\}$. Similarly define $V_{k}=$ $N \backslash \bigcup_{j=1}^{m(k)} S_{k}^{j}$ and let $M\left(V_{k}\right)=\left\{S_{k+1}^{1}, \ldots, S_{k+1}^{m(2)}\right\}$ for each $k$. Since $N$ is finite, this procedure terminates at some point (i.e., there is an integer $K$ such that

11 One does not need to use the full power of the weak top-coalition property to prove this result. As long as there exists a partition $\left\{S_{1}, \ldots, S_{K}\right\}$ such that $S_{k}$ is a weak topcoalition of $N \backslash\left(\bigcup_{m<k} S_{m}\right)$ for all $k$ (i.e., $S_{1}$ is a weak top-coalition of $N, S_{2}$ is a weak top-coalition of $N \backslash S_{1}$, and so on), the core is non-empty. 
$V_{K}=\varnothing$ and $\left.V_{K-1} \neq \varnothing\right)$. This procedure uniquely determines the coalition structure $\pi^{*}=\left\{S_{1}^{1}, \ldots, S_{1}^{m(1)}, S_{2}^{1}, \ldots, S_{2}^{m(2)}, \ldots, S_{K}^{1}, \ldots, S_{K}^{m(K)}\right\}$. We next show that $\pi^{*}$ is the only core partition. First note that a core partition should have $S_{1}^{j}$ as a component for any $j \in\{1, \ldots, m(1)\}$ since otherwise $S_{1}^{j}$ could profitably deviate. Given that, a core partition should have $S_{2}^{j}$ as a component for any $j \in\{1, \ldots, m(2)\}$, and so on. This completes the proof.

It is easy to see that the common ranking property implies the topcoalition property. However the converse relation does not hold. The following game makes this point:

Game 4. There are two type-b players and one type-a player. Thus, the player set $N$ can be written as $N=\left\{a_{1}, b_{1}, b_{2}\right\}$. Suppose that their preferences satisfy anonymity and let their preferences on cardinalities of coalitions be as follows:

Type a: $1 \succ_{a} 2 \succ_{a} 3$,

Type $b: 3 \succ_{b} 2 \succ_{b} 1$.

Claim. Game 4 satisfies the top-coalition property but not the common ranking property.

Proof of the Claim. First observe that if $a_{1} \in V$ then $\left\{a_{1}\right\}$ is a top coalition of $V$, and otherwise $V$ is a top-coalition of $V$. Thus, the top-coalition property is satisfied. On the other hand, the common ranking property is not satisfied: We have $\left\{a_{1}, b_{1}\right\} \succ_{a_{1}}\left\{a_{1}, b_{1}, b_{2}\right\}$ and yet $\left\{a_{1}, b_{1}, b_{2}\right\} \succ_{b_{1}}\left\{a_{1}, b_{1}\right\}$.

It is also clear that the top coalition property implies the weak topcoalition property. Example 3 in Section 6.6 shows that the converse relation does not hold.

The final issue that we investigate in this section is whether the top coalition properties imply the Scarf-balancedness condition in NTU games. An NTU game is a correspondence $V: 2^{N} \backslash\{\varnothing\} \mapsto \mathbb{R}^{N}$ such that: (i) for all $u, v \in$ $\mathbb{R}^{N},\left[\forall i \in S: u_{i}=v_{i}\right] \Rightarrow[u \in V(S) \Leftrightarrow v \in V(S)]$, (ii) $V(S)$ is closed, (iii) $V(S)$ $+\mathbb{R}_{-}^{N} \subset V(S)$ and (iv) there is an upper bound on the projection of $V(S)$ on $S$ for each $S \in 2^{N} \backslash\{\varnothing\}$. A balanced family of coalitions $\mathscr{B}$ is a collection of non-empty subsets of $N$ such that there is a (balanced weight) function $\delta: \mathscr{B} \rightarrow[0,1]$ with $\sum_{S \in \mathscr{B}, i \in S} \delta(S)=1$ for any $i \in N$. An NTU game $V$ is Scarf-balanced (Scarf 1967) if for every balanced family $\mathscr{B}$ of $N$ we have $\bigcap_{S \in \mathscr{B}} V(S) \subseteq \bigcup_{\pi \in \Pi(N)} \cap_{T \in \pi} V(T)$ where $\bigcup_{\pi \in \Pi(N)} \cap_{T \in \pi} V(T)$ denotes the superadditive cover of $V$.

Game 5. $N=\{1,2,3\}$, and the preferences are as follows:

$$
\begin{array}{ll}
\text { Player 1: } & \{1,2\} \succ_{1}\{1,3\} \succ_{1}\{1,2,3\} \succ_{1}\{1\}, \\
\text { Player 2: } & \{1,2\} \succ_{2}\{2,3\} \succ_{2}\{1,2,3\} \succ_{2}\{2\}, \\
\text { Player 3: } & \{1,3\} \succ_{3}\{2,3\} \succ_{3}\{1,2,3\} \succ_{3}\{3\} .
\end{array}
$$

Claim. Game 5 satisfies the common ranking property (and thus, both topcoalition properties as well) but not the Scarf-balancedness condition. 
Proof of the Claim. In this game we have the following common ranking:

$$
\{1,2\} \succ\{1,3\} \succ\{2,3\} \succ\{1,2,3\} \succ\{1\} \succ\{2\} \succ\{3\} \text {. }
$$

Thus, the common ranking property is satisfied. Now, we construct an NTU game from our game by appropriately assigning vector values to each coalition (see Shapley and Scarf 1973 and Quint and Shubik 1998):

$$
\begin{aligned}
& V(\{i\})=\left\{x \in \mathbb{R}^{3}: x_{i} \leq 0\right\} \text { for each } i \in N \\
& V(\{1,2\})=\left\{x \in \mathbb{R}^{3}: x_{1} \leq 3, x_{2} \leq 3\right\}, \\
& V(\{1,3\})=\left\{x \in \mathbb{R}^{3}: x_{1} \leq 2, x_{3} \leq 3\right\}, \\
& V(\{2,3\})=\left\{x \in \mathbb{R}^{3}: x_{2} \leq 2, x_{3} \leq 2\right\}, \text { and } \\
& V(\{1,2,3\})=\left\{x \in \mathbb{R}^{3}: x_{1} \leq 1, x_{2} \leq 1, x_{3} \leq 1\right\} .
\end{aligned}
$$

Next we check whether this NTU game is balanced. Observe that $\mathscr{B}=$ $\{\{1,2\},\{2,3\},\{1,3\}\}$ is a balanced family with a balanced weight $1 / 2$ for each coalition. Moreover $(2,2,2) \in \bigcap_{S \in \mathscr{B}} V(S)$. However, $(2,2,2) \notin \bigcup_{\pi \in \Pi(N)}$ $\cap_{S \in \pi} V(S)$. In other words, there is no partition that yields the payoff vector $(2,2,2)$. Thus, the game $V$ is not Scarf-balanced.

Although the weak top-coalition property is demanding, it is satisfied in many interesting applications. We present several such applications.

\section{Applications}

\subsection{Anonymous and separable games}

In Sects. 2 and 3 we discussed anonymity and additive separability. Here, we impose these two conditions together. (Note that separability together with anonymity implies additive separability). In such games the top-coalition property is satisfied and hence the core is non-empty. ${ }^{12}$

Corollary 1. Suppose that the game $G$ is anonymous and separable. Then it has a non-empty core.

Proof. We classify players using anonymity: Let $N^{+}=\left\{i \in N:\{i, j\} \succeq_{i}\{i\}\right.$ for any $j \neq i\}$ and $N^{-}=\left\{i \in N:\{i, j\} \prec_{i}\{i\}\right.$ for any $\left.j \neq i\right\}$. By definition $N^{+} \cup N^{-}=N$ and $N^{+} \cap N^{-}=\varnothing$. For any player $i \in N^{-}$, the top choice is staying alone by separability. Let $V \subseteq N$. If $V \cap N^{-} \neq \varnothing$ then any singleton subset of $V \cap N^{-}$is a top coalition in $V$. Otherwise $V \subseteq N^{+}$and $V$ itself is a top coalition in $V$. Thus, the top-coalition property is satisfied and $G$ has a core partition $\pi=\left\{N^{+},(\{i\})_{i \in N^{-}}\right\}$.

12 The common ranking property is not necessarily satisfied for such games. See Game 4 for an example. 


\subsection{Residential segregation with snob effects}

There are $K$ income classes $\{1,2, \ldots, K\}$ where each class $k$ has $n_{k}>0$ agents. Class 1 is the richest, class 2 is the second richest, and so on. Agents can form exclusive jurisdictions (coalitions). Each jurisdiction can be described by a list $\left(m_{1}, m_{2}, \ldots, m_{K}\right)$ that specifies the number of agents in each coalition. All agents have an identical utility function

$$
u\left(m_{1}, m_{2}, \ldots, m_{K}\right)=\frac{\sum_{k=1}^{K} w_{k} m_{k}}{\sum_{k=1}^{K} m_{k}}
$$

where $w_{1}>w_{2}>\cdots>w_{K}>0$ are weights of different income classes. That is, everyone wants to live in a rich jurisdiction. Coalition formation games induced by such problems satisfy the top-coalition property: For each $V \subseteq N$ members of its richest class is always a top coalition. Therefore a core partition exists. Moreover there is no mixed coalition at any core partition. Equivalently, perfect segregation occurs. ${ }^{13}$

\subsection{Becker's (1973) marriage game}

Let $M=\left\{m_{1}, m_{2}, \ldots, m_{n}\right\}$ be the set of men and $W=\left\{w_{1}, w_{2}, \ldots, w_{n}\right\}$ be the set of women. All men have identical preferences: they like $w_{1}$ the best, $w_{2}$ the second, ..., and $w_{n}$ the last. All women have identical preferences in an analogous way. For simplicity, we assume that staying single is the last choice for all agents. Becker (1973) suggests that $m_{i}$ and $w_{i}$ should be matched for any $i=1,2, \ldots, n$.

We can generate Becker's proposition as the unique core partition of the corresponding coalition formation game where players $m_{i} \in M$ and $w_{i} \in W$ have the following preferences:

$$
\begin{aligned}
& \left\{m_{i}, w_{1}\right\} \succ_{m_{i}}\left\{m_{i}, w_{2}\right\} \succ_{m_{i}} \cdots \succ_{m_{i}}\left\{m_{i}, w_{n}\right\} \succ_{m_{i}}\left\{m_{i}\right\} \succ_{m_{i}} \text { (others), } \\
& \left\{w_{i}, m_{1}\right\} \succ_{w_{i}}\left\{w_{i}, m_{2}\right\} \succ_{w_{i}} \cdots \succ_{w_{i}}\left\{w_{i}, m_{n}\right\} \succ_{w_{i}}\left\{w_{i}\right\} \succ_{w_{i}} \text { (others). }
\end{aligned}
$$

This game satisfies the top-coalition property. That is, for any non-empty coalition $V \subseteq N$ there is a top coalition: If $V \cap M \neq \varnothing$ and $V \cap W \neq \varnothing$ then $\left\{m_{i}, w_{j}\right\} \subseteq V$ is a top coalition, where $k \geq i$ for any man $m_{k} \in V$ and $\ell \geq j$ for any woman $w_{\ell} \in V$. That is because, $m_{i}$ and $w_{j}$ are the most attractive man and woman in $V$ respectively. If on the other hand $V \cap W=\varnothing$ or $V \cap M=\varnothing$, then every singleton subset of $V$ is a top coalition. By Theorem 2 the coalition structure $\pi^{*}=\left\{\left\{m_{1}, w_{1}\right\},\left\{m_{2}, w_{2}\right\}, \ldots,\left\{m_{n}, w_{n}\right\}\right\}$ is the unique core partition.

\subsection{TU Games with proportional sharing}

Let $N$ be a finite set of players and $v: 2^{N} \backslash\{\varnothing\} \rightarrow \mathbb{R}_{+}$be a real-valued function. The pair $(N, v)$ is called a $T U$ game and for any $S \subseteq N, v(S)$ is called the

${ }^{13}$ Rose-Ackerman (1977) analyzes a two class economy with exclusion that generates residential segregation. 
worth of coalition $S$. Suppose that the worth of each coalition is allocated among its members based on the proportional rule for some given list of shares $\left(\alpha_{i}\right)_{i \in N}$. Therefore when a coalition $S$ forms, the payoff to its member $i \in S$ is

$$
u_{i}(S)=\frac{\alpha_{i}}{\sum_{j \in S} \alpha_{j}} \times v(S)
$$

Example 1. Consider a village where the main industry is sugar cane production. Land is common property and it is in abundance. Each farmer $i \in N$ owns $\alpha_{i}>0$ units of labor. Sugar cane can be produced via labor using a common production function $f: \mathbb{R}_{+} \rightarrow \mathbb{R}_{+}$. Market price of sugar cane is normalized to one. Therefore the worth of coalition $S$ is $v(S)=f\left(\sum_{j \in S} \alpha_{j}\right)$. Finally, the worth of a coalition is shared among its members proportionally to labor inputs.

Note that, any TU game together with a proportional rule naturally induces a coalition formation game. This game satisfies the common ranking property and hence its core is non-empty. ${ }^{14}$

Corollary 2. Consider a simple coalition formation game $G$ that is induced by a proportional rule implemented on a TU game. Game G has a non-empty core.

Proof. Consider player $i \in N$. For any $S, T \in S_{i}(N)$ with $S \neq T$,

$$
\begin{aligned}
S \succeq_{i} T & \Leftrightarrow \frac{\alpha_{i}}{\sum_{j \in S} \alpha_{j}} \times v(S) \geq \frac{\alpha_{i}}{\sum_{j \in T} \alpha_{j}} \times v(T) \\
& \Leftrightarrow \frac{v(S)}{\sum_{j \in S} \alpha_{j}} \geq \frac{v(T)}{\sum_{j \in T} \alpha_{j}}
\end{aligned}
$$

Define $\varphi(S)=\frac{v(S)}{\sum_{j \in S} \alpha_{j}}$. For any $i \in N$ and any $S, T \in S_{i}(N), S \succeq_{i} T$ iff $\varphi(S) \geq \varphi(T)$. We next show that there is a top coalition for each $V \subseteq N$ with $V \neq \varnothing$. Let $M(V)=\left\{S \in 2^{V} \backslash\{\varnothing\}: \varphi(S) \geq \varphi(T)\right.$ for any $\left.T \in 2^{V} \backslash\{\varnothing\}\right\}$. Each element $S$ of $M(V)$ is a top coalition. This is because, for any $i \in S$ and any $T \in 2^{V} \backslash\{\varnothing\}$ we have $\varphi(S) \geq \varphi(T)$ or equivalently $S \succeq_{i} T$. Thus, the common ranking property (and therefore the top-coalition properties as well) is satisfied. Theorem 1 implies that there is a core partition.

We can cover Farrell and Scotchmer's (1988) partnership game and Moulin and Shenker's (1994) average cost sharing problem as special cases of TU games with proportional sharing.

\subsubsection{Farrell and Scotchmer's (1988) partnership game}

Let $N=\{1,2, \ldots, n\}$ and each player $j \in N$ has ability $e_{j}>0$. The productivity of each coalition $S \in 2^{N} \backslash\{\varnothing\}$ is $t(|S|) \times \sum_{j \in S} e_{j}$, where $t: \mathbb{N} \rightarrow \mathbb{R}_{+}$.

\footnotetext{
14 Jackson and Wolinsky's (1996) technique to prove existence of a stable network is an application of this argument. See also Dutta and Mutuswami (1997).
} 
When a coalition $S$ forms, its output is shared equally among its members and hence the payoff to each member is

$$
\frac{t(|S|) \times \sum_{j \in S} e_{j}}{|S|} .
$$

Farrell and Scotchmer's partnership games is a specific application of TU games with proportional sharing where $v(S)=t(|S|) \times \sum_{j \in S} e_{j}$ for all $S \in N$ and $\left(\alpha_{i}\right)_{i \in N}=(1,1, \ldots, 1)$. Therefore partnership games satisfy the common ranking property (as was shown in Farrell and Scotchmer 1988) and thus it has a non-empty core.

\subsubsection{Moulin and Shenker's (1994) average cost sharing problem}

Let $N=\{1,2, \ldots, n\}$ and each player $i \in N$ be endowed with divisible money. There is one divisible commodity besides money which can be produced by money with a common production technology that is accessible by anybody or any coalition. The technology is described by a cost function $C: \mathbb{R}_{+} \rightarrow \mathbb{R}_{+}$ that is a mapping from the amount of commodity production to the amount of money needed. Each player $i \in N$ has a fixed demand $a_{i}>0$ for the commodity. Players have monotonic preferences on money. Players are allowed to form coalitions and produce the commodity jointly using the common production technology. For a non-empty coalition $S \subseteq N$, amount of production needed is $\sum_{j \in S} a_{j}$ and therefore the cost of production is $C\left(\sum_{j \in S} a_{j}\right)$. The cost is shared based on the average cost sharing rule. That is, each player in a coalition pays the average cost of the coalition times her demand. Moulin and Shenker's average cost sharing problem is another application of TU games with proportional sharing where $v(S)=-C\left(\sum_{j \in S} a_{j}\right)$ for all $S \subseteq N$ and $\left(\alpha_{i}\right)_{i \in N}=\left(a_{i}\right)_{i \in N}$. Therefore a coalition formation game that is induced by the average cost sharing rule satisfies the common ranking property and thus has a non-empty core.

\subsection{Shapley and Scarf's (1974) housing market}

Let $N=\{1,2, \ldots, n\}$ and each player $i \in N$ own one indivisible house $h_{i}$. Thus, the set of houses can be denoted by $H(N)=\left\{h_{1}, h_{2}, \ldots, h_{n}\right\}$. Similarly, we can define $H(V)$ for any $V \subseteq N$ with $V \neq \varnothing$. Each player $i$ can consume (or live in) at most one house, and has a strict preferences $P_{i}$ over $H(N)$. An allocation is a one-to-one and onto mapping $\mu: N \rightarrow H(N)$. A weak coalitional deviation from $\mu$ is a pair $(S, v)$ such that (i) $S \subseteq N$ with $S \neq \varnothing$, (ii) $v$ : $S \rightarrow H(S)$ is one-to-one and onto, and (iii) $v(i) P_{i} \mu(i)$ for at least one $i \in S$ and $v(i)=\mu(i)$ for others. A strict core allocation is an allocation $\mu$ that is immune to any weak coalitional deviation. Shapley and Scarf (1974) show that the core is non-empty and Roth and Postlewaite (1977) show that the strict core is singleton and coincides with the unique competitive allocation.

David Gale invented the following top trading cycles algorithm that yields the competitive allocation: Pick any $i \in N$ and let her point her most favorite house in $H(N)$, say house $h_{j}$. If $i=j$ then $i \rightarrow i$ is a top cycle. Otherwise, $j \neq i$ 
and let $j$ point her most favorite house, say $h_{k}$. If $k=i$ then $i \rightarrow j \rightarrow i$ is a top cycle and if $k=j$ then $j \rightarrow j$ is a top cycle. Otherwise let $k$ point her most favorite house, say $h_{\ell}$. If $\ell=i$ then $i \rightarrow j \rightarrow k \rightarrow i$ is a top cycle, if $\ell=j$ then $j \rightarrow k \rightarrow j$ is a top cycle, and if $\ell=k$ then $k \rightarrow k$ is a top cycle. Otherwise, let $\ell$ choose her most favorite house, and so on and so forth. Since the number of players is finite, by continuing this procedure we find at least one trading cycle. Let the set of players in the first top trading cycle be $S_{1}^{*}$. Remove them out of the market together with their assignments. We now have a reduced group of players $V_{1}=N \backslash S_{1}^{*}$ and houses $H\left(V_{1}\right)$. Among the remaining players $V_{1}$, there is a subset $S_{2}^{*}$ that forms a top cycle. Again remove them from the market together with their assignments and proceed in a similar way. This algorithm yields the unique competitive allocation that coincides with the unique strict core allocation. Given any housing market, the competitive mechanism naturally induces a coalition formation game: For any non-empty subset of players $V$, let $\mu_{V}^{*}: V \rightarrow H(V)$ be the unique competitive allocation. Then, for any player $i$ and any two coalitions $S, T \in S_{i}(N)$, we have $S \succeq_{i} T$ $\Leftrightarrow \mu_{S}^{*}(i) R_{i} \mu_{T}^{*}(i)$. By the dynamics of the top trading cycles algorithm $S_{1}^{*} \in$ $M(N)$, and $S_{k}^{*} \in M\left(V_{k}\right)$ for each $k$ with $V_{k} \neq \varnothing$. Therefore the top-coalition property is satisfied for the induced coalition formation game. Note that the common ranking property is not necessarily satisfied for such games.

Example 2. $N=\{1,2,3\}$ and the preferences are as follows:

$$
\begin{aligned}
& h_{2} \succ_{1} h_{3} \succ_{1} h_{1}, \\
& h_{3} \succ_{2} h_{1} \succ_{2} h_{2}, \\
& h_{2} \succ_{3} h_{1} \succ_{3} h_{3} .
\end{aligned}
$$

Let $V=\{1,2\}$. We have

$$
\mu_{N}^{*}=\left(\begin{array}{ccc}
1 & 2 & 3 \\
h_{1} & h_{3} & h_{2}
\end{array}\right) \quad \text { and } \quad \mu_{V}^{*}=\left(\begin{array}{cc}
1 & 2 \\
h_{2} & h_{1}
\end{array}\right) .
$$

Therefore $\mu_{V}^{*}(1) P_{1} \mu_{N}^{*}(1)$ and $\mu_{N}^{*}(2) P_{2} \mu_{V}^{*}(2)$ which implies $V \succ_{1} N$ and $N \succ_{2} V$ violating the common ranking property.

\subsection{Benassy's (1982) uniform reallocation}

Let $N=\{1,2, \ldots, n\}$ and each player $i \in N$ be endowed with an initial endowment $\omega(i) \in \mathbb{R}_{+}$of a perfectly divisible commodity. Each player $i$ has a single-peaked preference relation $R_{i}$ over $\mathbb{R}_{+}$. That means she has a best consumption $b(i) \in \mathbb{R}_{+}$such that: for all $x, y \in \mathbb{R}_{+},[x<y \leq b(i)$ or $x>y \geq b(i)]$ $\Rightarrow y P_{i} x$. Whenever $z(i)=b(i)-\omega(i) \geq 0$ we say agent $i$ has an excess demand and whenever $z(i)=b(i)-\omega(i) \leq 0$ we say she has an excess supply. Similarly, a coalition $V \subseteq N$ has an excess demand if $z(V)=\sum_{i \in V} z(i) \geq 0$ and it has an excess supply if $z(V)=\sum_{i \in V} z(i) \leq 0$. For any coalition $V \subseteq N$, a feasible allocation is a vector $x \in \mathbb{R}_{+}^{V}$ such that $\sum_{i \in V} x(i)=\sum_{i \in V} \omega(i)$. When a coalition $V \subseteq N$ forms, its members reallocate $\omega(V)=\sum_{i \in V} \omega(i)$ based on 
the uniform reallocation rule: For all $i \in V$,

$$
U_{V}(i)= \begin{cases}\min \{b(i), \omega(i)+\lambda\} & \text { if } z(V) \geq 0 \\ \max \{b(i), \omega(i)+\lambda\} & \text { if } z(V) \leq 0\end{cases}
$$

where $\lambda \in \mathbb{R}$ solves $\sum_{i \in V} U_{V}(i)=\omega(V)$.

A detailed analysis of the uniform reallocation rule is beyond the scope of this paper. ${ }^{15}$ However the following feature of its is of particular importance to us: Whenever the coalition $V$ has an excess demand, the uniform rule $U_{V}$ allocates (i) each agent $i \in V$ who has an excess supply, her best consumption $b(i)$ and (ii) each agent $i \in V$ who has an excess demand, at least her endowment $\omega(i)$. Similarly whenever the coalition $V$ has an excess supply, the uniform rule $U_{V}$ allocates (i) each agent $i \in V$ who has an excess demand, her best consumption $b(i)$ and (ii) each agent $i \in V$ who has an excess supply, at most her endowment $\omega(i)$. This feature implies that, whenever all agents have excess demand (or whenever all have excess supply), the uniform rule allocates them their endowments.

For any given triple $\left(N,(\omega(i))_{i \in N},\left(R_{i}\right)_{i \in N}\right)$, the uniform reallocation rule induces a coalition formation game: For any player $i \in N$, and any two coalitions $S, T \in S_{i}(N)$, we have $S \succeq_{i} T \Leftrightarrow U_{S}(i) R_{i} U_{T}(i)$. This game satisfies the weak top-coalition property and hence has a non-empty core.

Corollary 3. Consider a coalition formation game $G$ that is induced by the uniform reallocation rule. Game G has a non-empty core.

Proof. We show that, for any $V \subseteq N$ the coalition $V$ itself is a weak topcoalition. Suppose without loss of generality that coalition $V$ has an excess demand $z(V) \geq 0$ (the other case is similar). Define $V^{1}=\{i \in V: z(i) \leq 0\}$ and $V^{2}=\{i \in V: z(i)>0\}$. We have two cases to consider.

Case 1. $V^{1} \neq \varnothing$. Let $i \in V^{1}$. The uniform rule $U_{V}$ allocates agent $i$ her best consumption $b(i)$. Therefore, for all $T \subseteq V$ we have $V \succeq_{i} T$. Next consider an agent $i \in V^{2}$ and suppose $T \subseteq V$ is such that $T \succ_{i} V$. We will show that $T \cap V^{1} \neq \varnothing$ which in turn implies that $V$ is a weak top-coalition. The uniform rule $U_{V}$ allocates agent $i$ at least her endowment $\omega(i)$. Since $z(i)=$ $b(i)-\omega(i)>0$ and the preferences are single-peaked, the relation $T \succ_{i} V$ implies $U_{T}(i)>U_{V}(i) \geq \omega(i)$. But that means, there is at least one agent $j \in T$ with $z(j)<0$ since otherwise the uniform rule $U_{T}$ allocates all agents in $T$ (including agent $i$ ) their endowments. Hence $T \cap V^{1} \neq \varnothing$ and $V$ is a weak top-coalition with $\left\{V^{1}, V^{2}\right\}$ being the associated partition.

Case 2. $V^{1}=\varnothing$. In this case all agents in $V$ are excess demanders. Therefore no matter which subcoalition $T \subseteq V$ forms, all its members are also excess demanders and the uniform rule $U_{T}$ allocates each member her endowment. As a result all agents in $V$ are indifferent between all subsets of $V$. Hence $V$ is

15 Sprumont (1991) initiated a large literature that analyzes the uniform rule. See also Ching (1992, 1994), Thomson (1994a, 1994b, 1995) and Klaus et al. (1998). 
a top coalition (and thus a weak top-coalition as well). Therefore the weak top-coalition property is satisfied and the coalition structure $\{N\}$ is a core partition.

Finally note that, the top coalition property is not necessarily satisfied in these coalition formation games.

Example 3. $N=\{1,2,3\},(\omega(1), \omega(2), \omega(3))=(5,5,5)$, and $(b(1), b(2), b(3))$ $=(1,8,8)$. The following table gives the outcome of the uniform rule $U_{T}$, for any $T \subseteq N$ :

\begin{tabular}{|l|c|c|c|}
\hline & 1 & 2 & 3 \\
\hline$U_{\{1\}}$ & 5 & - & - \\
\hline$U_{\{2\}}$ & - & 5 & - \\
\hline$U_{\{3\}}$ & - & - & 5 \\
\hline$U_{\{1,2\}}$ & 2 & 8 & - \\
\hline$U_{\{1,3\}}$ & 2 & - & 8 \\
\hline$U_{\{2,3\}}$ & - & 5 & 5 \\
\hline$U_{\{1,2,3\}}$ & 1 & 7 & 7 \\
\hline
\end{tabular}

We have

$$
\begin{array}{ll}
N \succ_{1} T \quad \text { for all } T \subseteq N \text { with } 1 \in T, \\
\{1,2\} \succ_{2} T \quad \text { for all } T \subseteq N \text { with } 2 \in T, \\
\{1,3\} \succ_{3} T \quad \text { for all } T \subseteq N \text { with } 3 \in T,
\end{array}
$$

and therefore there is no top coalition for the grand coalition $N$.

\section{References}

Alcalde J (1995) Exchange-proofness or divorce-proofness? Stability in one-sided matching markets. Econ Design 1: 275-287

Becker G (1973) A theory of marriage, Part I. J Polit Econ 81: 813-846

Benassy JP (1982) The economics of market equilibrium. Academic Press, San Diego

Bogomolnaia A, Jackson MO (1998) The stability of hedonic coalition structures. mimeo

Ching S (1992) A simple characterization of the uniform rule. Econ Letters 40: 57-60

Ching S (1994) An alternative characterization of the uniform rule. Soc Choice Welfare 11: $131-136$

Demange G (1982) Single-peaked orders on a tree. Math Soc Sci 3: 389-396

Demange G (1994) Intermediate preferences and stable coalition structures. J Math Econ 23: 45-58

Dutta B, Mutuswami S (1997) Stable networks. J Econ Theory 76: 322-344 
Dréze J, Greenberg J (1980) Hedonic coalitions: Optimality and stability. Econometrica 48: 987-1003

Farrell J, Scotchmer S (1988) Partnerships. Q J Econ: 279-297

Gale D, Shapley L (1962) College admissions and the stability of marriage. Am Math Mon 69: 9-15

Greenberg J (1977) Pure and local public goods: A game-theoretic approach. In: Sandmo A (ed.) Public finance, Heath and Co, Lexington, MA

Greenberg J, Weber S (1986) Strong Tiebout equilibrium under restricted preference domain. J Econ Theory 38: 101-117

Greenberg J, Weber S (1993) Stable coalition structures with unidimensional set of alternatives. J Econ Theory 60: 62-82

Guesnerie R, Oddou C (1981) Second best taxation as a game. J Econ Theory 25: 6791

Jackson M, Wolinsky A (1996) A Strategic model of social and economic networks. J Econ Theory 71: 44-74

Kaneko M, Wooders MH (1986) The core of a game with a continuum of players and finite coalitions; the model and some results. Math Soc Sci 12: 105-137

Klaus B, Peters H, Storcken T (1998) Strategy-proof division with single-peaked preferences and individual endowments. Soc Choice Welfare 15: 297-311

Milchtaich I, Winter E (1997) Stability and segregation in group formation. mimeo

Moulin H, Shenker S (1994) Average cost pricing versus serial cost sharing: An axiomatic comparison. J Econ Theory 64: 178-201

Quint T, Shubik M (1998) Games of status and one-to-one ordinal preference games. Working Paper, Cowles Foundation, University of Nevada

Rose-Ackerman S (1977) The political economy of a racist housing market. J Urban Econ 4: 150-169

Roth A, Postlewaite A (1977) Weak versus strong domination in a market with indivisible goods. J Math Econ 4: 131-137

Roth A, Sotomayor M (1990) Two-sided matching: A study in game-theoretic modeling and analysis. Cambridge University Press, New York

Scarf H (1967) The core of an $N$ person game. Econometrica 35: 50-69

Shapley L, Scarf H (1974) On cores and indivisibility. J Math Econ 1: 23-37

Sprumont Y (1991) The division problem with single-peaked preferences: A characterization of the uniform allocation rule. Econometrica 59: 509-519

Tan JJM (1991) A necessary and sufficient condition for the existence of a complete stable matching, J Algorithm 12: 154-178

Thomson W (1994) Consistent solutions to the problem of fair division when preferences are single-peaked. J Econ Theory 63: 219-245

Thomson W (1994) Resource-monotonic solutions to the problem of fair division when preferences are single-peaked. Soc Choice Welfare 11: 205-223

Thomson W (1995) Population-monotonic solutions to the problem of fair division when preferences are single-peaked. Econ Theory 5: 229-246

Weber S, Zamir S (1985) Proportional taxation: Non-existence of stable structures in an economy with public good. J Econ Theory 35: 178-185 